\title{
Central airway stenosis in a patient with autoimmune pancreatitis
}

\author{
M. Ito*, M. Yasuo*, H. Yamamoto*, K. Tsushima*, T. Tanabe*, T. Yokoyama*, \\ H. Hamano ${ }^{\#}$, S. Kawa", T. Uehara ${ }^{+}$, T. Honda ${ }^{+}$, S. Kawakami ${ }^{\S}$ and K. Kubo*
}

ABSTRACT: Autoimmune pancreatitis is a unique form of chronic pancreatitis characterised by a high-serum immunoglobulin (Ig)G4 concentration involving various extra pancreatic lesions.

A 63-yr-old female with autoimmune pancreatitis complained of cough. Chest computed tomography revealed an irregular stenosis of the central airway, lung hilar and mediastinal lymph node swelling, and a marked thickness of the bronchovascular bundle. Bronchoscopic examination revealed an irregular tracheobronchial stenosis accompanied with an oedematous mucosa and engorged vessels. Lung hilar and mediastinal lymph node swelling, central airway stenosis and bronchoscopic findings remarkably resembled those of sarcoidosis.

Bronchial biopsy specimens demonstrated diffuse infiltrations of plasma cells, lymphocytes and eosinophils with fibrosis. Immunostaining showed infiltration of several IgG4-positive plasma cells. The patient was treated with oral prednisolone at $1 \mathrm{mg} \cdot \mathrm{kg}^{-1} \cdot \mathrm{day}^{-1}$ for pancreatic lesions. A month later, the lung lesions, including central airway stenosis, lung hilar and mediastinal lymph node swelling, and bronchovascular bundle thickness, had dramatically improved along with improvement of pancreatitis, thus indicating a close association between the two conditions.

This is the first report of a patient with autoimmune pancreatitis showing central airway stenosis similar to that of sarcoidosis.

\section{KEYWORDS: Airway, autoimmune disease, bronchoscopy, immunopathology}



utoimmune pancreatitis is a unique form of chronic pancreatitis characterised by a high-serum immunoglobulin (Ig)G4 concentration and various extra pancreatic complications, including sclerosing sialoadenitis, sclerosing cholangitis, retroperitoneal fibrosis, tubulointerstital nephritis [1] and lung lesions, such as hilar lymphadenopathy, lung nodule [2] and interstitial pneumonia [3]; thus suggesting that this disease consists of a spectrum of systematic diseases. These extra pancreatic lesions exhibit similar histopathological findings to those in the pancreas, including dense infiltration of IgG4-positive plasma cells and T lymphocytes with fibrosis [1], and have sometimes been misdiagnosed as the specific diseases of corresponding organs, such as Sjoegren's syndrome for lachrymal and salivary gland lesions and primary sclerosing cholangitis for bile duct lesion, leading to incorrect therapy [4]. Herein, the present authors report a patient with autoimmune pancreatitis showing central airway stenosis in which special care was needed to differentiate it from sarcoidosis.

\section{CASE REPORT}

A 63-yr-old female was admitted to the present authors' hospital (Shinshu University School of Medicine, Matsumoto, Japan) complaining of cough, a loss of appetite and pale stool. She had undergone resection of a right submandibular gland neoplasm at age 58 yrs. The peripheral blood cell count was normal and a biochemistry examination revealed the following: total bilirubin $0.49 \mathrm{mg} \cdot \mathrm{dL}^{-1}$; alanine aminotransferase $77 \mathrm{IU} \cdot \mathrm{L}^{-1}$; aspartate aminotranferase $28 \mathrm{IU} \cdot \mathrm{L}^{-1}$; alkaline phosphatase $425 \mathrm{U} \cdot \mathrm{L}^{-1}$ (normal range: 124-367 U. $\mathrm{L}^{-1}$ ); $\gamma$-glutamyltransferase $127 \mathrm{IU} \cdot \mathrm{L}^{-1}$ (normal range: 6$30 \mathrm{IU} \cdot \mathrm{L}^{-1}$ ); and amylase $48 \mathrm{IU} \cdot \mathrm{L}^{-1}$ (normal range: $\left.44-127 \mathrm{IU} \cdot \mathrm{L}^{-1}\right)$. The IgG level was elevated to $2,889 \mathrm{mg} \cdot \mathrm{dL}^{-1}$. At the same time, the patient was diagnosed with diabetes. Other serological tests were within the normal range. Radiological studies showed diffuse enlargement of the pancreas, a circumference of lymphadenopathy and an irregular narrowing of the main pancreatic duct; these findings corresponded to those of autoimmune pancreatitis. Serum IgG4 was measured and

\section{AFFILIATIONS}

*First Dept of Internal Medicine,

\# Second Dept of Internal Medicine, +Dept of Laboratory Medicine,

${ }^{\S}$ Dept of Radiology, Shinshu

University School of Medicine, and

- Center for Health, Safety and

Environmental Management, Shinshu

University, Matsumoto, Japan.

\section{CORRESPONDENCE}

M. Yasuo

First Dept of Internal Medicine

Shinshu University School of

Medicine

3-1-1 Asahi

Matsumoto 390-8621

Japan

Fax: 81263363722

E-mail: yasumasa@shinshu-u.ac.jp

Received:

April 032008

Accepted after revision:

September 172008

STATEMENT OF INTEREST

None declared. 
revealed an elevated value of $1,660 \mathrm{mg} \cdot \mathrm{dL}^{-1}$. An immunohistochemical examination of biopsy specimens from the bile duct showed infiltration of IgG4-positive plasma cells. Therefore, the patient was diagnosed to have autoimmune pancreatitis.

The left submandibular gland swelling and lung hilar and mediastinal lymph node swelling were detected by chest computed tomography (CT) and thought to be the extra pancreatic lesions of autoimmune pancreatitis. In addition, the patient had suffered from cough and chest CT revealed an irregular stenosis of the central airway and a marked thickness of the bronchovascular bundle (fig. 1a). A lung function test revealed a vital capacity of $3.11 \mathrm{~L}$ (129\% predicted), forced expiratory volume in one second (FEV1) of $2.17 \mathrm{~L}(106.4 \%$ pred), FEV1/forced vital capacity (FVC) of $67.8 \%$ and a diffusing capacity of the lung for carbon monoxide of $17.7 \mathrm{~mL} \cdot \mathrm{min}^{-1} \cdot \mathrm{mmHg}^{-1}(84.3 \%$ pred $)$.

Bronchoscopic examination unveiled an irregular tracheobronchial stenosis accompanied with mucosal oedema and engorged vessels (fig. 2), which closely mimicked the findings observed in sarcoidosis. Analysis of the bronchoalveolar lavage fluid revealed a cell count of $4.0 \times 10^{4} \cdot \mathrm{mL}^{-1}$, with $60.6 \%$ macrophages, 39\% lymphocytes (CD4/8 ratio 4.48 ), $0 \%$ eosinophils and $0.4 \%$ neutrophils, which were also similar to the findings of sarcoidosis. Bronchial biopsy specimens demonstrated diffuse inflammatory infiltrates consisting mainly of plasma cells, lymphocytes and scattered eosinophils with fibrosis.

An immunohistochemical examination showed infiltration of several IgG4-positive plasma cells (fig. 3). The number of IgG4positive cells was superior to that of IgG1-positive cells, and the number identified per high power field (HPF) was scored as severe (>30 per HPF) according to KAMISAWA et al. [5], which has been described elsewhere [6]. The present authors did not identify any specific finding in the biopsy specimens from right S2 and S3. At this time, the serum angiotensin-converting enzyme (ACE) level was $5.3 \mathrm{U} \cdot \mathrm{I}^{-1} \quad\left(7.0 \sim 25.0 \mathrm{U} \cdot \mathrm{I}^{-1}\right)$. These findings were similar to the findings of the bile duct and were considered to be an IgG4-related change. The patient was treated with oral prednisolone at $1 \mathrm{mg} \cdot \mathrm{kg}^{-1} \cdot \mathrm{day}^{-1}$. One month later, the enlargement of the pancreas, the circumference of lymphadenopathy, the irregular narrowing of the main pancreatic duct and left submandibular gland swelling had all dramatically improved. A chest CT revealed a significant improvement of the central airway stenosis (fig. 1b), lung hilar and mediastinal lymph node swelling, and the thickness of the bronchovascular bundles. The IgG4 level decreased to $515 \mathrm{mg} \cdot \mathrm{dL}^{-1}$. The results of the lung function tests were also improved. The vital capacity was $3.23 \mathrm{~L}$ (134\% pred), FEV1 was $2.86 \mathrm{~L}$ (140.2\% pred), FEV1/FVC was $89.9 \%$ and diffusing capacity of the lung for carbon monoxide was $25.3 \mathrm{~mL} \cdot \mathrm{min}^{-1} \cdot \mathrm{mmHg}^{-1}(120.2 \%$ pred $)$.

\section{DISCUSSION}

Mediastinal lymphadenopathy [1, 2], interstitial pneumonia [3] and inflammatory pseudotumour of the lung $[3,7]$ have been previously reported as pulmonary manifestations of autoimmune pancreatitis; however, no pulmonary lesion of central airway stenosis has been reported. In previous studies, histological examinations of the lung lesions showed a dense infiltration of lymphocytes and plasma cells in the thickened interstitium of the alveoli, around the bronchioles and the peribronchovascular region. In addition, immunostaining showed an infiltration of IgG4-positive plasma cells in these areas [2, 3, 7]. ZEN et al. [7] reported an inflammatory pseudotumour with IgG4-positive plasma cells. They also observed an irregular narrowing of bronchioles entrapped in nodules and an interstitial pneumonia pattern at the boundaries of nodules. The present case showed the central airway stenosis and thickness of the bronchovascular bundles. In addition, the immunohistochemical findings of bronchial mucosal biopsy specimens were similar to those observed in previous studies, but with no special findings in bronchioles.


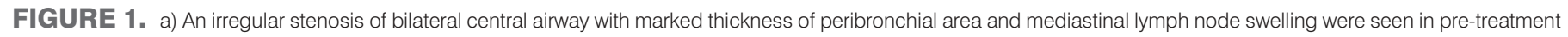
of chest computed tomography. b) An improvement of central airway stenosis and mediastinal lymph node swelling was seen after prednisolone treatment. 


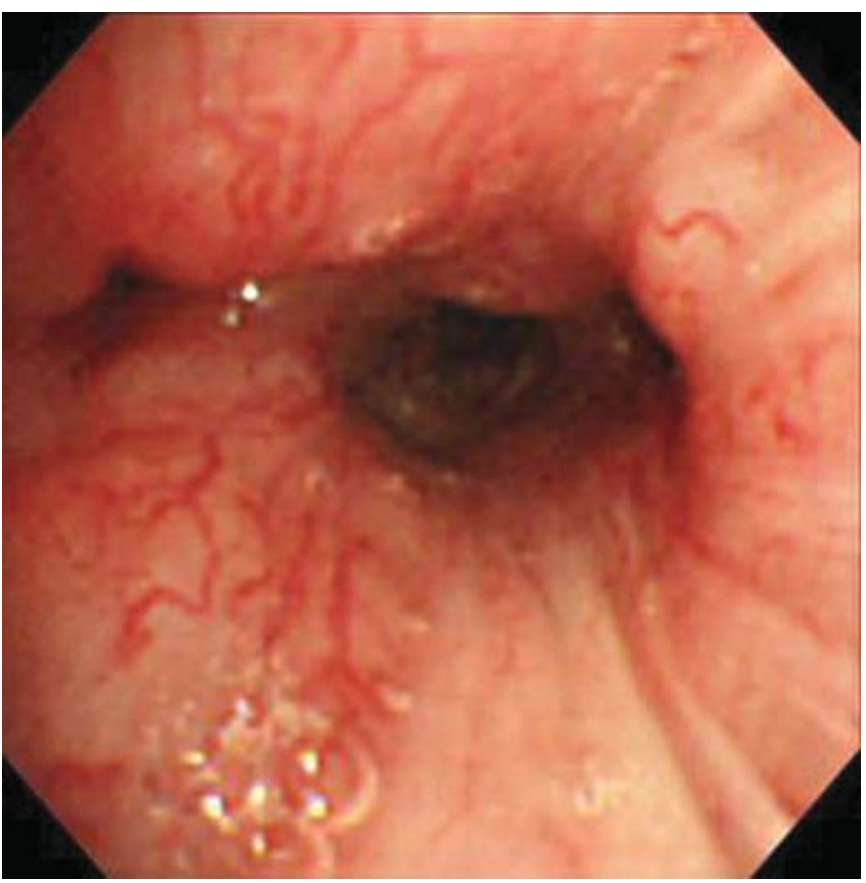

FIGURE 2. An irregular tracheobronchial stenosis accompanied with mucosal oedema and engorged vessels were seen by bronchoscopy.

Steroid therapy dramatically improved the central airway stenosis, thus indicating a close association between central airway stenosis and autoimmune pancreatitis. Accordingly, the pulmonary manifestations of central airway stenosis found in the present case are considered to be IgG4-related lesions. Lung lesions of autoimmune pancreatitis, including the interstitium of the alveoli, around the bronchioles, the peribronchovascular region and central airway, were all in the broad category of the interstitium.

Lung hilar and mediastinal lymph node swelling are characteristics of sarcoidosis and central airway stenosis has been reported to be one of the clinical phenotypes [8]. In sarcoidosis, granulomas and serum ACE elevation were often observed [9]; however, in the present case, granulomatous change was not detected in the central airway specimens and a normal ACE value was shown. In addition, the present authors did not observe any IgG4-positive plasma cells in the bronchial biopsy specimens of four sarcoidosis patients with a thickened bronchial wall who were examined at Shinshu University School of Medicine. Therefore, these clinical findings were able to help differentiate between IgG4-related pulmonary lesion and sarcoidosis. However, IgG4-related lesions have sometimes been found without any pancreatic manifestations. The solitary manifestation of an IgG4 related pulmonary lesion may show findings similar to those of sarcoidosis. Therefore, special care is required to accurately differentiate between these two conditions.

Some studies found high serum concentrations of IgG4 in patients with atopic dermatitis, asthma and some parasitic diseases, which are a direct response to an exogenous antigen [10]. Patients with severe asthma sometimes showed irregular tracheobronchial stenosis accompanied with an oedematous



FIGURE 3. An immunohistochemical examination of bronchial biopsy specimen shows dense infiltration of immunoglobulin G4-positive plasma cells in submucosal area. Scale bar $=50 \mu \mathrm{m}$.

change of the mucosa; however, the present patient never had any episodes of bronchial asthma. In addition, IgG4-positive cell infiltration has been found in pancreatic cancer and chronic pancreatitis [11, 12]. However, IgG4-positive cells in these diseases are fewer than in autoimmune pancreatitis patients and the tissue specimens of these cases were not associated with lymphoplasmacytic sclerosing pancreatitis, which was the characteristic attribute of autoimmune pancreatitis.

In conclusion, the present authors experienced an impressive case of central airway stenosis with autoimmune pancreatitis. The characteristic findings of this case were central airway stenosis, submucosal immunoglobulin G4 positive plasma cell involvement and an obvious relief of the stenosis by corticosteroids. As a result, such cases need to be carefully differentiated from sarcoidosis.

\section{REFERENCES}

1 Kawa S, Hamano H. Clinical features of autoimmune pancreatitis. J Gastroenterol 2007; 42: 9-14.

2 Hamed G, Tsushima K, Yasuo M, et al. Inflammatory lesions of the lung, submandibular gland, bile duct and prostate in a patient with IgG4-associated multifocal sysytemic fibrosis. Respirology 2007; 12: 455-457. 
3 Kobayashi H, Shimokawaji T, Kanoh S, Motoyoshi K, Aida S. IgG4-positive pulmonary disease. J Thorac Imaging 2007; 22: 360-362.

4 Kamisawa T, Okamoto A. Autoimmune pancreatitis: proposal of IgG4-related sclerosing disease. J Gastroenterol 2006; 41: 613-625.

5 Kamisawa T, Funata N, Hayashi Y, et al. A new clinicopathological entity of IgG4-related autoimmune disease. J Gastroenterol 2003; 38: 982-984.

6 Chari ST. Diagnosis of autoimmune pancreatitis using its five cardinal features: introducing the Mayo Clinic's HISORt criteria. J Gastroenterol 2007; 42: Suppl. 42, 39-41.

7 Zen Y, Kitagawa S, Minato H, et al. IgG4-positive plasma cells in inflammatory pseudotumor (plasma cell granuloma) of the lung. Human Pathol 2005; 36: 710-717.
8 Brandstetter RD, Messina MS, Spirince NL, Grillo HC. Tracheal stenosis due to sarcoidosis. Chest 1981; 80: 656.

9 Iannuzzi MC, Rybicki BA, Teirstein AS. Sarcoidosis. N Engl J Med 2007; 357: 2153-2165.

10 Hussain R, Poindexter RW, Ottesen EA. Control of allergic reactivity in human filariasis: predominant localization of blocking antibody to the IgG4 subclass. J Immunol 1992; 148: 2731-2737.

11 Ghazale A, Chari ST, Smyrk TC, et al. Value of serum IgG4 in the diagnosis of autoimmune pancreatitis and in distinguishing it from pancreatic cancer. Am J Gastroenterol 2007; 102: 1-8.

12 Zhang L, Notohara K, Levy MJ, Chari ST, Smyrk TC. IgG4positive plasma cell infiltration in the diagnosis of autoimmune pancreatitis. Modern Pathology 2007; 20: 23-28. 